\title{
1988 E-MRS Fall Meeting Highlights Superconductors, Particle Detectors, Magnets and Solid State Ionics
}

The second 1988 meeting of the European-Materials Research Society took place at its traditional venue, Strasbourg on the river Rhine, situated between West Germany and France. The conference itself, in the grand atmosphere of the European Parliament, attracted more than 400 participants, bringing the total number of 1988 E-MRS participants to well over 1,000 for the first time.

Four symposia were held:

- Symposium A-High Temperature Superconductors: Preparations, chaired by P.F. Bongers (Philips Research Labs, Eindhoven), C. Schenkler (LEPES-CNRS, Grenoble), and B. Stritzker (ISI-KFA, Juilich), which predictably attracted the largest fraction of audience attention;

- Symposium B-The Properties, Processing, and Applications of Rare Earth, IronRich, High Performance Magnets, chaired by I.R. Harris (Birmingham University);

- Symposium C-Superconducting and Low Temperature Particle Detectors, chaired by G. Waysand (University of Paris);

- Symposium D-Solid State Ionics, chaired by M. Balkanski (Université Pierre et Marie Curie).

The plenary lectures were given by K.H.J. Bushow (Eindhoven) and G. Deutscher (Tel Aviv).

Symposia A, B, and D are summarized below. Full-length refereed papers, both contributed and invited, will be published by Elsevier North-Holland as part of the EMRS Symposium Proceedings series.

\section{Symposium A-High Temperature Superconductors: Preparation and Applications}

Chairs: P.F. Bongers (Philips Research Laboratories, Eindhoven, Netherlands), C. Schlenker (LEPES, CNRS, Grenoble, France), and B. Stritzker (ISI, KFA, Jülich, West Germany).

Over 220 scientists from 25 countries attended this symposium. Thirteen invited papers and more than 130 contributions will be published in the Jourmal of Less Common Metals. The symposium was devoted mostly to the preparation and characterization of the new high $\mathrm{T}_{\mathrm{c}}$ superconducting ceramics, and single crystals and thin films of the Y-Ba-Cu-O series. However, theoretical aspects were also reviewed (B.K. Chakraverty, L. Gorkov, P. Peyral et al.).

Since the discovery of superconductivity in the $\mathrm{La}(\mathrm{Ba}, \mathrm{Sr}) \mathrm{CuO}$ system by Bednorz

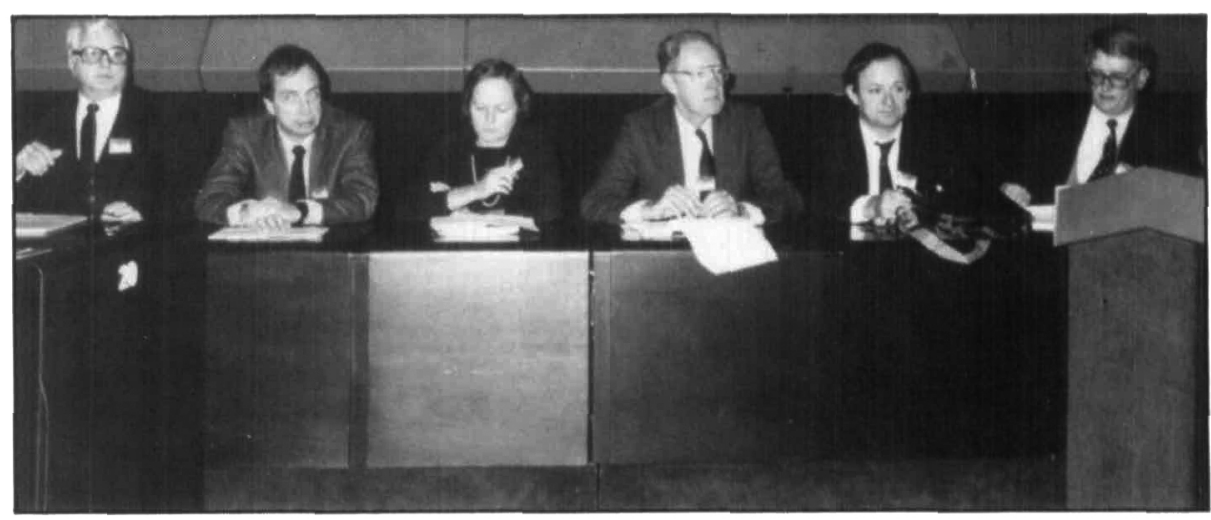

Chairs of the symposia at 1988 E-MRS Fall Meeting (left to right): M. Balkanski, B. Stritzker, C. Schlenker, P.F. Bongers, G. Waysand, and I.R. Harris.

and Müller, several other superconducting copper oxide compounds have appeared. Their structures are closely related since they can all be described as the stacking of $\mathrm{Cu}$-containing perovskite blocks (usually oxygen deficient) separated by blocks having different types of atomic arrangement. According to D.M. de Leeuw, the ground state of all these oxides is insulating but the presence of itinerant holes in the $\mathrm{CuO}_{2}$ planes created by doping leads to superconductivity. Depending on the nature of the inserted blocks, different homologous series or families of phases are formed: $\mathrm{YBa}_{2} \mathrm{Cu}_{3} \mathrm{O}_{6+1}, \mathrm{Bi}_{2} \mathrm{Sr}_{2} \mathrm{Ca}_{n .1} \mathrm{Cu}_{n} \mathrm{O}_{4+2 n}, \mathrm{Tl}_{2} \mathrm{Ba}_{2}$ $\mathrm{Ca}_{n .1} \mathrm{Cu}_{n} \mathrm{O}_{4,2 n}, \mathrm{TlBa}_{2} \mathrm{Ca}_{n, 1} \mathrm{Cu}_{n} \mathrm{O}_{2 \mathrm{n}+2.5}$ etc. It appears that $T_{c}$ increases with $n$ for $n \leqslant 3$, then decreases for $\geqslant 4$.

The most unexpected and perhaps the most important news came from R.J. Cava, who reported the discovery of a new family of superconducting copper oxides: $\mathrm{Pb}_{2} \mathrm{Sr}_{2} \mathrm{LnCu}_{3} \mathrm{O}_{8+.}$. In this case the inserted block is formed by two $\mathrm{PbO}$ planes separated by a single $\mathrm{CuO}$, layer. In these compounds, superconductivity can be induced by partial substitution of the rare earth $(\mathrm{Ln})$ or $\mathrm{Y}$ by $\mathrm{Ca}$. The highest transition temperature $\left(\mathrm{T}_{\mathrm{c}} \approx 68 \mathrm{~K}\right)$ was reached for $\mathrm{Pb}_{2} \mathrm{Sr}_{2} \mathrm{Y}_{0.5} \mathrm{Ca}_{0.5} \mathrm{Cu}_{3} \mathrm{O}_{4}$. Production of this phase is very sensitive to the preparation conditions (temperature and oxygen partial pressure) because of the oxidation of $\mathrm{Pb}^{2+}$ into $\mathrm{Pb}^{4+}$. Ceramics as well as single crystals were obtained.

In bismuth and thallium phases, the effects of cation substitution have been studied by different groups. For example, the substitution of $\mathrm{Cu}$ by $\mathrm{Fe}$ in the $\mathrm{Bi}$ phases does not change the superstructure but destroys superconductivity. Several groups (S. Amelinckx et al., L. Pierre et al.) reported partial substitution of the bismuth cations by lead cations in these phases. This substitution leads to an increase of $T$. in a given phase. It also helps to increase the proportion of the phase having the highest $\mathrm{T}_{\mathrm{c}}$. A new superstructure appears, probably due to $\mathrm{Bi} / \mathrm{Pb}$ order. Depending on the thermal treatment of the samples the order is long or short range.

In the thallium compounds also, preparation conditions have a large influence on structural and physical properties. P. Bordet et al. reported that $\mathrm{Tl}_{2} \mathrm{Ba}_{2} \mathrm{CuO}_{6}$ can be either orthorhombic and nonsuperconducting or tetragonal (disordered) and superconducting. In both cases neutron diffraction studies have shown the presence of thallium and oxygen vacancies. Thallium vacancies were also observed in $\mathrm{Tl}_{2} \mathrm{Ba}_{2} \mathrm{CaCu}_{2} \mathrm{O}_{8}$ but not if $\mathrm{Ca}$ is replaced by $\mathrm{Y}$ or a rare earth. If $\mathrm{Ba}$ is replaced by $\mathrm{Sr}$ and $\mathrm{Tl}$ by $\mathrm{Pb}$ simultaneously a new superstructure appears and the crystals have the same lamellar morphology as $\mathrm{Bi}$ compounds, probably due to the presence of $\mathrm{Pb}^{2+}$ lone pair (M. Hervieu et al.).

Another type of inserted block appears in $\mathrm{Y}_{2} \mathrm{Ba}_{4} \mathrm{Cu}_{8} \mathrm{O}_{16}(2-4-8)$ which was first discovered as thin films by A. Kapitulnik et al. E. Kaldis et al. reported its preparation under pressure as ceramics or single crystals and the structure determination by powder neutron diffraction. The cornersharing infinite square chains of $\mathrm{YBa}_{2} \mathrm{Cu}_{3} \mathrm{O}_{7}(1-2-3)$ are replaced by double edge-sharing square chains. The main in- 
terest of this compound $\left(T_{c} \approx 80 \mathrm{~K}\right)$ comes from the thermal stability of its oxygen content, which may make it more suitable for applications. The 1-2-3.5 compound $\left(T_{c} \approx\right.$ $40 \mathrm{~K}$ ) is intermediate between the 1-2-3 and 2-4-8 compounds and it is possible that other compounds having other stacking sequences exist in this family.

After this brief review of the different superconducting copper oxides phases, it is worth mentioning the real need for single crystals for the characterization and study of physical properties of these compounds. For example, the use of $\mathrm{YBa}_{2} \mathrm{Cu}_{3} \mathrm{O}_{7}$ single crystals in NMR and $N Q R$ studies have allowed detection of the two different $\mathrm{Cu}$ sites ( $\mathrm{P}$. Segransan et al.). In oxygen deficient samples $\left(\mathrm{YBa}_{2} \mathrm{Cu}_{3} \mathrm{O}_{6.75}\right)$ the presence of $\mathrm{Cu}^{1+}$ in the $\mathrm{Cu}-\mathrm{O}$ chains has been demonstrated and the almost perfect order of oxygen vacancies proposed by M.A. Alario et al. from electron diffraction has been confirmed.

Unfortunately, according to H.J. Scheel, up till now, crystals available have not been really good single crystals. For $\mathrm{YBa}_{2} \mathrm{Cu}_{3} \mathrm{O}_{7}$, impurities even at very low concentrations $(<1 \%)$ have an effect on crystal growth (for example, $\mathrm{Al}$ impurities slow down the kinetics of oxidation) and also create defects in the structure. Both these effects influence the physical properties. Consequently, the choice of the crucible is very important in order to prevent contamination.

The crystal growth of $\mathrm{Bi}$ and $\mathrm{Tl}$ compounds and even their synthesis as ceramics is even more difficult than for $\mathrm{YBa}_{2} \mathrm{Cu}_{3} \mathrm{O}$, since no phase diagram exists yet. However, the best way to obtain crystals in the Bi system consists in using a strong temperature gradient followed by slow cooling.

Thin film studies are almost exclusively devoted to $\mathrm{YBa}_{2} \mathrm{Cu}_{3} \mathrm{O}_{7}$. The deposition methods mainly used are electron beam co-evaporation, RF magnetron sputtering and laser evaporation. The most commonly used substrates are $\mathrm{SrTiO}_{3}, \mathrm{MgO}$, $\mathrm{Al}_{2} \mathrm{O}_{3}, \mathrm{ZrO}_{2}$. Films are usually made by a two-step method including post-annealing in $\mathrm{O}_{2}$ flow. The annealing temperature and process are very critical to the production of the superconducting phase U. Geerk et al., B. Dam et al., C. Escribe-Filippini et al.).

One of the most important difficulties in fabricating thin films is to avoid substrate reaction and diffusion. $\mathrm{Bi}-\mathrm{Sr}-\mathrm{Ca}-\mathrm{Cu}-\mathrm{O}$ films have been characterized by RBS and ERDA techniques (E. Fogarassy et al., F. Rochet et al.). Y-Ba-Cu-O films on $\mathrm{MgO}$ or $\mathrm{Al}_{2} \mathrm{O}_{3}$ have been studied by scanning Auger microscopy and Angle Resolved XPS (E.G. Keim et al.). For $\mathrm{Al}_{2} \mathrm{O}_{3}$ substrates, $\mathrm{Ba}$ aluminate forms at the interface and grain boundaries; this is the main cause of high electrical resistance. For $\mathrm{MgO}$ substrates, the large $\mathrm{Cu}$ diffusion at the interface induces Cu loss, which leads to a decrease of $\mathrm{T}_{\mathrm{r}}$ and consequently to the degradation of film quality. These degradation processes occur mainly during the $\mathrm{O}_{2}$ post annealing. Therefore, methods of direct production for high $T_{c}$ superconducting thin films without post annealing (U. Poppe et al., J. Froehlingsdorf et al.) are of great interest. Y-Ba-Cu-O films on $\mathrm{SrTiO}_{3}$ and $\mathrm{MgO}$ have been produced by pure oxygen DC sputtering.

In order to use high $T_{c}$ superconductors in microelectronic devices, it is necessary to find a process compatible with device fabrication technology. With this in mind, H.U. Habermeier et al. explored plasma oxidation in order to keep the specimen temperature as low as $80^{\circ} \mathrm{C}$. Others have been able to obtain thin films on piezoelectric substrates $\left(\mathrm{LiNbO}_{3}\right)$ or $\mathrm{Si}$, which may be suitable for technical applications $(\mathrm{H}$. Schmidt et al., J. Tate et al.). It is also important to note that the $\mathrm{Y}_{2} \mathrm{Ba}_{4} \mathrm{Cu}_{8} \mathrm{O}_{16}$ films ob- tained by $\mathrm{A}$. Kapitulnik et al. have better characteristics than classical $\mathrm{YBa}_{2} \mathrm{Cu}_{3} \mathrm{O}_{7}$ ones: same $J_{c}\left(>210^{7} \mathrm{~A} / \mathrm{cm}^{2}\right.$ at $\left.4.2 \mathrm{~K}\right)$ but lower resistivity in the normal state.

Concerning the physical properties, some interesting results have been reported on flux pinning and flux creep both on bulk and on thin films samples ( $L$. Fruchter et al., R. Griessen et al., R.G. Humphreys et al.).

In applications, high $T_{c}$ oxides currently seem to be better candidates for squid devices (R.H. Koch et al., C.E. Gough) and high frequency applications $(M$. Hein et al.) rather than wires and cables due to the low critical currents, even though current densities of up to $5,000 \mathrm{~A} / \mathrm{cm}^{2}$ in tapes made by Hitachi have been reported by $R$. Flikiger.

More and more superconducting oxides phases have been discovered recently. However, it is clear from this conference that, at the same time, their preparation becomes more difficult to obtain both pure phases and good single crystals. Consequently, the production of wellcharacterized good quality materials is

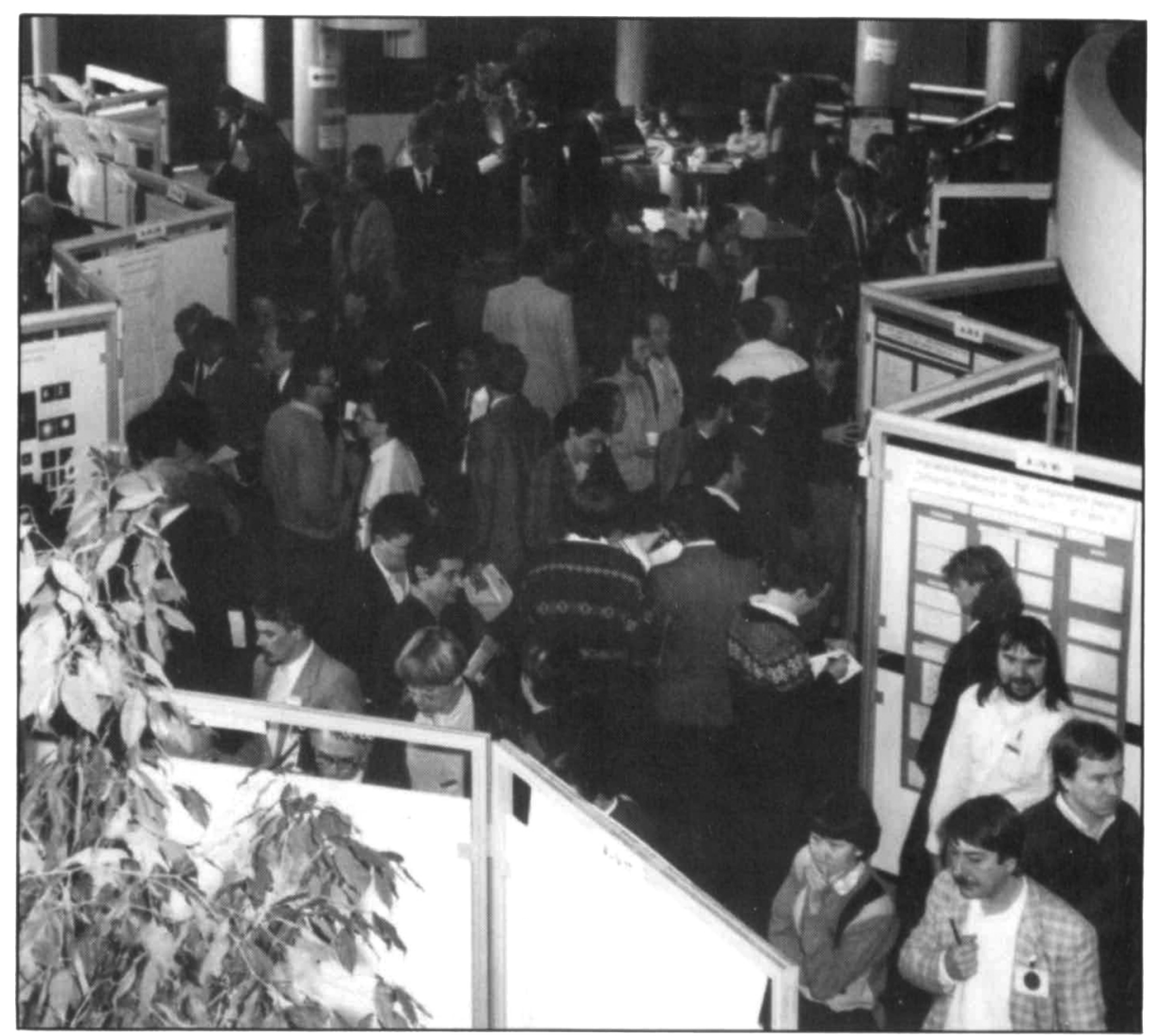

Poster session at the 1988 E-MRS Fall Meeting draws a large audience. Review speakers were assigned to critically assess posters in their areas of expertise, and they led a stimulating three-hour discussion of the posters during a final session. 
fundamental. It is clear that studies of the physical properties will give reliable results only when performed on such samples. This requires increased collaboration between the chemists who prepare the samples and those who characterize from both a structural and physical point of view.

C. Chaillout Laboratoire de Cristallographie CNRS

\section{Symposium B-Iron-Rich High Performance Permanent Magnets}

Chair: I.R. Harris (Metallurgy \& Materials, University of Birmingham, United Kingdom).

The object of this meeting was to bring together physicists, materials scientists, and electrical engineers to discuss aspects of advanced permanent magnets and to obtain an up-to-date appraisal of their status.

The meeting began with a plenary lecture from Prof. Buschow (Philips Eindhoven), who gave an interesting and wide ranging talk on the physics and applications of novel magnetic materials. $\mathrm{He}$ considered the $\mathrm{R}_{2} \mathrm{Fe}_{14} \mathrm{~B}$-type materials and the isomorphous $\mathrm{R}_{2} \mathrm{Fe}_{14} \mathrm{~B}$ and $\mathrm{R}_{2} \mathrm{Co}_{14} \mathrm{~B}$ compounds. In addition he described new ternary compounds based on the ThM $n_{12}$-type structure. His lecture included a description of permanent magnet fabrication together with some technological applications.

During the poster session, some 24 posters covered a wide range of topics encompassing fundamental physics, microstructure, processing, and applications. There was a very high standard of presentation. Review speakers were assigned to critically assess the posters in their areas of expertise so they could then lead a discussion of the posters at the end of the final session.

The session on November 10 began with a talk by Coey (Trinity College Dublin) on the intrinsic properties of compounds with the $\mathrm{Nd}_{2} \mathrm{Fe}_{14} \mathrm{~B}$ structure. Factors such as the anisotropy field were considered and the potential of new compounds was assessed. This talk was followed by a contribution from Givord (CNRS Grenoble) on coercivity mechanisms in Nd-Fe-B-type magnets, including sintered, melt-spun and hot-worked varieties. Experimental results were compared with various theoretical predictions.

Harris (University of Birmingham) described the processing of $\mathrm{Nd}-\mathrm{Fe}-\mathrm{B}$-type magnets and illustrated the versatile nature of the Nd-Fe-B-type alloys with regard to processing. He dealt with the various powder routes and with casting and hot working methods. Tokunaga's (Hitachi) talk on the processing of melt-spun material included a description of the production of isotropic and anisotropic magnets. The beneficial influence of gallium additions on the magnetic properties was described and discussed. The last talk of the morning was given by Henig (MPL Stuttgart), who outlined the phase relationships and microstructure in the Nd-Fe-B-type alloys. The influences of such elements as oxygen and aluminum were described and discussed.

The afternoon session began with Fidler (TU Vienna), who described the electron microscopy of $\mathrm{Nd}$-Fe-B-type magnets and this talk nicely complemented the earlier contributions. Emphasis was placed on the complex nature of the grain boundary regions and on the distribution of minor alloy additions such as aluminum, which is known to markedly influence the coercivity by modifying the grain boundaries. The last speaker, Hanitsch (TU Berlin), reviewed the application of Nd-Fe-B-type magnets to electromagnetic machines. He dealt with the relationships between the magnetic properties (such as B) and a particular application. The talks ended on a high note with an expansive view of the future of these magnets in a variety of applications.

This session was followed by a vigorous three-hour discussion period, during which invited speakers reviewed the posters. Each area was the subject of stimulating discussion with nearly all the participants making a contribution. The format devised for this meeting worked well and the program gave ample opportunity for everyone to contribute.

As a result of these discussions it became clear that there is still much work to be done in order to understand the detailed relationships between microstructure and coercivity in these materials. All in all it was a stimulating meeting. The magneticians left Strasbourg in high spirits and, 1 hope, full of good ideas for future research into these fascinating materials.

I.R. Harris

\section{Symposium D-Solid State Ionics}

Chair: M. Balkanski (Laboratoire de Physique des Solides, Université Pierre et Marie Curie, Paris)

M. Balkanski's plenary talk reviewed the recent advances in solid state ionics which are opening the way to micro-ionics. Developments in fast ion conductors and cathodic materials useful as mixed ionic and electronic conductors lead to their foreseeable application in solid state microbatteries, microsensors and optical devices. This symposium gave an overview of the experimental and theoretical aspects of the physics of these materials, and of the technological problems involved in making the microdevices. Over 50 papers were presented in 10 sessions, including 8 invited papers, 25 oral contributions, and 12 posters.

The first session was opened by $A$. Levasseur (Université de Bordeaux I, Talence), who focused on the solid state batteries realized to date, with glassy, polymeric or crystalline materials as the electrolyte and with insertion compounds or amorphous solids as cathodes. Subsequent papers emphasized the promising applications of thin film batteries in devices requiring low current densities. The main concerns were different techniques for growing cathodic materials, such as molecular beam deposition, RF sputtering, and chemical vapor deposition. Research results on the electrical properties of these compounds were presented.

G. Meunier (Université de Bordeaux I) described a solid state microbattery using a new titanium compound as the positive electrode (obtained by RF sputtering), the ternary sputtered oxide glass $\left(\mathrm{B}_{2} \mathrm{O}_{3}-\mathrm{Li}_{2} \mathrm{O}\right.$ $\mathrm{Li}_{2} \mathrm{SO}_{4}$ ) as the electrolyte, and evaporated lithium as the negative anode.

C. Poinsignon (Ecole Nationale Superieure d'Electrochimie et $D^{\prime}$ Electrometallurgie, Saint Martin d'Heres) reviewed the most common polymer-electrolytes and their main conduction mechanisms.

One session was dedicated to the glassy materials. After an introductory talk by $\mathrm{M}$. Ribes (Université des Science et Techn. du Languedoc, Montpellier) on the theory of ionic conductivity in glassy materials, papers on the structural and transport properties of lithium borate glasses were presented.

The next three sessions focused on investigations of the structural, electronic and optical properties of layered compounds in the pure and intercalated phases. R. Brec and G. Ouvrard (Institut de Physique et Chimie des Materiaux, Nantes) illustrated the shift of metal cation occurring in transition metal chalcogenides upon lithium intercalation, which produces a progressive amorphization of the cathodes during the charge-discharge cycles. C. Delmas (Universite de Bordeaux I, Talence) covered the properties of layered oxides. W.Y. Liang (University of Cambridge, U.K.) discussed the electronic properties of intercalation compounds, emphasizing the nature of the acceptor levels in the host material and on the bands structure modifications induced by the charge transfer mechanism following the intercalation process.

P.A. Burret (Université Pierre et Marie Curie, Paris) proposed the shift of the sec- 
ond excitonic peak upon intercalation as a result of resonant Raman scattering measurements carried out on indium selenide. Raman spectroscopy can also provide a tool for monitoring the variation of the Néel temperature upon lithium intercalation in iron phosphorus trisulfide. This possibility is based on appearance of new Raman lines produced by folding of the phonon branches associated with the antiferromagnetic phase, as illustrated in the paper by T. Sekine (Université Pierre et Marie Curie, Paris).

Y. Chabre (Université Joseph Fourier, Grenoble) discussed the information obtainable by NMR in the study of charge transfer, ion mobility, and structural modifications of insertion compounds. He also illustrated the feasibility of a special solid state electrochemical cell in order to perform in situ NMR measurements.

Diffusion theory was discussed in the following session by R. Elliott (Department of Theoretical Physics, Oxford). Elliott reviewed the theory of hopping atomic diffusion in crystals, including the correlation effect due to the blocking of sites available for hopping by other atoms and the correlation effects introduced by the forces between diffusing particles. Methods for decoupling the effective equations of motion were proposed. An example of the extension of this formalism to the diffusion in a disordered medium was discussed in a subsequent paper by R. Brak (Department of Theoretical Physics, Oxford).

Lattice dynamics calculations were presented for different systems relevant in solid state ionics. M. Kanehisa (Université Pierre et Marie Curie, Paris) investigated within a Bethe lattice model the vibrations of borate glass, revealing that certain molecular modes evolve into extended modes when the infinite lattice is formed, while certain modes remain localized. $M$. Bernasconi (Universitá di Milano) presented the lattice dynamics of lithium-

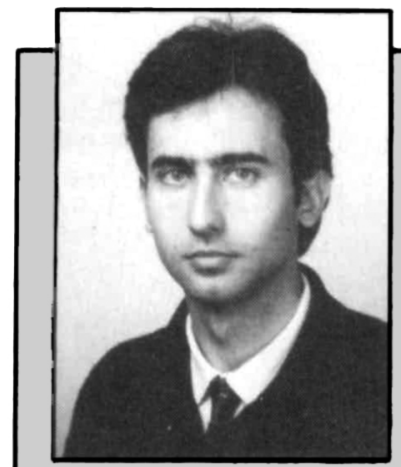

M. Bemasconi

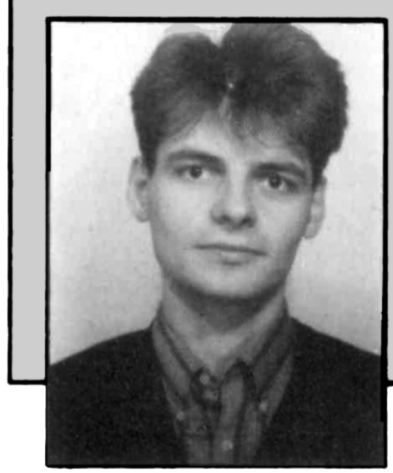

Awands Presented for Best Papers

Marco Bernasconi and Pierre Alain Burret received awards from E-MRS in recognition of the quality of the work presented in their papers given in the Symposium on Solid State Ionics.

Bernasconi was recognized for his presentation on "Lattice Dynamics of Lithium Intercalated FePS3." A Doctor in Physics at the University of Milan, he is currently involved with research in the dynamics of layered crystals and the dynamics of mixed semiconductors (AlGaAs), and he has several publications in this area to his credit.

Burret was recognized for his presentation on "The Effects of Lithium Intercalation on the Optical Properties of InSe." He is finishing his PhD dissertation at the University of Paris under the direction of Prof. M. Balkanski. Using techniques such as infrared and far infrared spectroscopy and Raman scattering, Burret has been able to investigate the influence of lithium on the electronic levels of the host, intraband transitions that accompany any charge transfer, and the plasmonphonon coupling during a semiconductor-metal transition.

P.A. Burret

intercalated iron phosphorus trisulfide, predicting a sensible shift of certain phonon peaks in the Raman spectra useful for monitoring the lithium concentration. M. Balkanski (Université Pierre et Marie Curie) interpreted the reflectivity spectra of lithium conducting borate glasses on the basis of a microscopic model in which the lithium ions move in a double-well potential.

The final session focused on electrochromic materials. The electrochromism effect is a persistent but reversible optical change produced electrochemically, by applying an electrical field or current to a suitable material. A. Donnadieu (Université des Science et Techn. du Languedoc, Montpellier) reviewed the state-of-the-art in this field. The papers in this session covered recent investigations on viologens, polymeric, and insertion compounds.

In his closing address Prof. Balkanski presented an E-MRS award for the best papers of the symposium to Marco Bernasconi for his presentation on "Lattice Dynamics of Lithium Intercalated FePS3" and to Pierre Alain Burret for his presentation on "The Effects of Lithium Intercalation on the Optical Properties of InSe."

Marco Bernasconi University of Milan

Need information about the European Materials Research Society... E-MRS membership, upcoming meetings and proceedings?

Contact:

P. Siffert, E-MRS President

Centre de Recherches Nucléaires

Laboratoire PHASE

67037 Strasbourg, Cedex, France

Telephone (88) 286543 